\title{
Stress Kills
}

Berhane Worku ${ }^{1}$, shudhansu alishetti ${ }^{2}$, and kumudha ramasubba ${ }^{3}$

${ }^{1}$ New York Presbyterian Weill Cornell Medicine

${ }^{2}$ New York-Presbyterian Brooklyn Methodist Hospital

${ }^{3}$ Affiliation not available

November 25, 2020

Stress Kills

Berhane Worku MD ${ }^{1}$, Shudhanshu Alishetti ${ }^{2}$, Kumudha Ramasubbu ${ }^{2}$

1. New York Presbyterian Brooklyn Methodist Hospital/Weill Cornell Medical Center Department of Cardiothoracic Surgery, Brooklyn, NY 11215

2. New York Presbyterian Brooklyn Methodist Hospital Division of Cardiology, Brooklyn NY 11215

Corresponding Author

Berhane Worku MD

Department of Cardiothoracic Surgery

New York Presbyterian Brooklyn Methodist Hospital

$5066^{\text {th }}$ Street

Brooklyn, NY 11215

The medical, economic, and social consequences of the COVID-19 pandemic have been profound. Severe respiratory failure as well as inflammatory and thrombotic complications have resulted in hundreds of thousands of deaths. Political controversy continues regarding optimal strategies for large scale control of the pandemic. Social distancing policies have led to reduced transmission rates but the economic effects have been devastating. Optimal treatment strategies continue to evolve, and vaccine solutions are on the horizon. In addition to these more obvious issues, other severe consequences of the pandemic are slowly being recognized.

In the current report, Kir et. al. describe two postmenopausal women presenting with signs and symptoms of acute coronary syndrome in the setting of severe psychological stress related to social isolation during the COVID-19 pandemic (1). Both were COVID negative and both had unremarkable coronary angiograms. Both were diagnosed with takotsubo cardiomyopathy based on the characteristic findings of angina, mild troponin elevation, electrocardiographic changes, and apical akinesis on echocardiogram. Both admitted to severe anxiety and stress in the days prior to the onset of symptoms. Both improved with conservative management including beta-blockers and anxiolytics with resolution of apical akinesis on follow up echocardiogram.

Takotsubo or stress cardiomyopathy is a now well recognized entity typically presenting as angina or dyspnea in the setting of a severe emotional or physical stressor. Postmenopausal women are more frequently affected and a history of psychiatric disorders is frequently noted. Electrocardiographic abnormalities and mild troponin elevations are common. Diagnosis is based on the InterTAK diagnostic score. Echocardiography 
classically demonstrates apical ballooning with basal hyperkinesis, but other wall motion abnormalities are described usually extending beyond a traditional coronary artery distribution. Coronary angiography is frequently performed to rule out acute coronary syndrome but is unremarkable. The syndrome is typically self-limited, requiring conservative supportive management, but in severe cases can lead to heart failure and shock requiring high-dose pharmacologic support, mechanical circulatory support, and in $\sim 5 \%$ of cases can be fatal (2).

Emotional and physical stress are risk factors for a variety of conditions including cardiovascular disease. Furthermore, psychiatric disorders such as depression and anxiety are associated with poorer outcomes in the setting of cardiovascular disease. Proposed mechanisms for this include behavioral factors such as noncompliance with medications and lifestyle modifications (diet, exercise, smoking cessation). Biological factors are also suggested, including altered autonomic nervous system activity with elevations in catecholamine levels and inflammatory responses amongst others (3). Similar hypotheses have been put forth regarding the mechanism of takotsubo stress cardiomyopathy and perhaps some overlap exists between the cardiovascular manifestations of psychiatric disorders and overt stress cardiomyopathy.

The COVID-19 pandemic has had several medical consequences beyond those related to viral infection itself. The suspension of certain medical and surgical services potentially allows for the natural history of various diseases to take their course. Unemployment impairs the ability of many to access what medical services remain available. Psychiatric disorders are inflamed in the setting of social, economic, and other stressors. A four to five-fold increase in the incidence of stress cardiomyopathy has been noted during the months following the COVID-19 outbreak unrelated to COVID-19 infection itself, presumably the consequence of stress related to the abovementioned effects of the pandemic and our response to it. (4). COVID-19 has taught us that stress kills.

\section{REFERENCES}

1. Kir D, Beer N, De Marchena EJ. Takutsobo cardiomyopathy caused by emotional stressors in the Coronavirus Disease 2019 (COVID-19) pandemic era. J Card Surg in press

2. De Chazal HM, Del Buono MG, Keyser-Marcus L, Ma L, Moeller FG, Berrocal D, Abbate A. Stress cardiomyopathy diagnosis and treatment. J Am Coll Cardiol 2018;72:1955-71

3. Takagi H, Ando T, Umemoto. Perioperative depression or anxiety and postoperative mortality in cardiac surgery: a systematic review and meta-analysis. Heart Vessels 2017;32:1458-1468

4. Jabri A, Kalra a, Kumar A, Alameh A, Adroja S, Bashir H, Nowacki AS, Shah R, Khubber S, Kanaa'N A, Hedrick DP, Sleik KM, Mehta N, Chung MK, Khot UN, Kapadia SR, Puri R, Reed GW. Incidence of stress cardiomyopathy during the coronavirus disease 2019 pandemic. JAMA Netw Open 2020 Jul 1;3(7):e2014780. doi: 10.1001/jamanetworkopen.2020.14780 